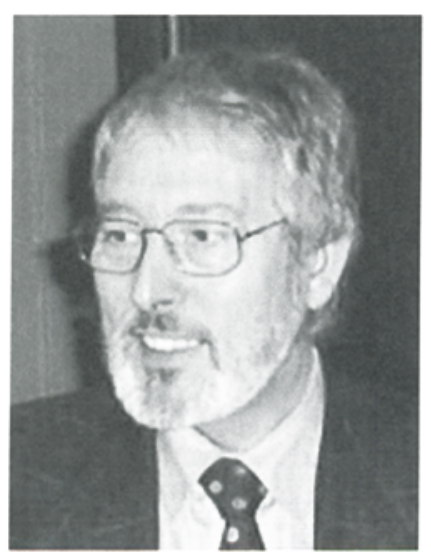

Rolf Harbeck

Gasteditor dieses Heftes

\section{Topographie und Kartographie -}

so hat die Arbeitsgemeinschaft der Vermessungsverwaltungen der Länder der Bundesrepublik Deutschland (AdV) 1996 einen ihrer Facharbeitskreise benannt und damit die ganzheitliche Sichtweise der Erdoberflächenbeschreibung als Aufgabe der Landesvermessung zum Ausdruck gebracht. Dass hierbei digitale Verfahren und Modelle im Vordergrund stehen, ist das Ergebnis einer Entwicklung, die 1985 mit der ATKIS-Idee eingesetzt hat.

Digitale Landschaftsmodelle, Digitale Geländemodelle, Digitale Topographische Karten und Digitale Orthophotos sind heute die vier Produktbereiche, mit denen die Landesvermessung die geotopographische Infrastruktur für Deutschland bereit stellt. Folgerichtig hat die AdV 2002 ihren diesbezüglichen Arbeitskreis in "Geotopographie" und ihren Arbeitskreis "Grundlagenvermessung" in "Raumbezug" umbenannt.

Das vorliegende, der Landesvermessung gewidmete Heft der KN, richtet den Blick des Lesers auf einige konzeptionelle Arbeitsergebnisse der AdV, die von den Ländern in Dienste und Produkte umgesetzt werden.

$H$. Derenbach und A. Schleyer stellen den Satellitenpositionierungsdienst SAPOS ${ }^{R}$ vor und zeigen seine Bedeutung für die moderne Kartographie auf. J. Zahn gibt einen Abriss über den Stand der Entwicklungs- und Produktionsarbeiten an den kartographischen ATKIS ${ }^{R}$-Komponenten.

Auch die klassische Topographische Karte 1:50000, selbstverständlich in digitaler, rasterformatierter, interaktiver Form, findet Berücksichtigung:

K. Tönnessen berichtet über die von der $A d V$ initiierte, erfolgreiche CD-ROMSerie Top50, über ihre privaten und professionellen Anwendungsmöglichkeiten und über die vorgesehenen Weiterentwicklungen.
In diesem Zusammenhang darf ein Blick auf 3D- und Virtual Reality-Anwendungen im Internet nicht fehlen. A. Riedl aus Wien schildert uns Entwicklung, Trends und Perspektiven der Web3D-Technologien; sie liefern mit ihren Standards und Formaten die Basis, mit deren Hilfe 3D/VR-Anwendungen, vom Modellieren über das Rendering bis hin zur Ausgabe, im Internet umgesetzt werden können. Durch die hohe Nachfrage in diesem Marktsegment ist es in letzter Zeit zu interessanten Neupositionierungen gekommen, die in diesem Aufsatz einer kritischen Bewertung unterzogen werden.

Kartographie als Veranschaulichung von Geodaten - dieser Aufgabe widmen sich die AdV und ihr Facharbeitskreis "Geotopographie" auch weiterhin. Technische Regelwerke, z.B. zur Geodatenaktualität, zur Struktur von Orthophotos, zur Metadatenkatalogisierung oder zur objekt- und signaturenbasierten Landschaftsmodellierung, werden entwickelt und zu AdV-Standards erhoben. Fachliche Recherchen und technisch-wissenschaftliche Konzepte werden erarbeitet und, wie im Falle des ATKIS ${ }^{\circledR}$-Projekts Modell- und kartographische Generalisierung, von den Ländern zur Verfahrensentwicklung herangezogen. Festzuhalten bleibt, dass die moderne topographische Kartographie - zunehmend auch in ziviler und militärischer Zusammenarbeit - ein fester Bestandteil der Landesvermessung als einer Säule des amtlichen Vermessungswesens bleiben wird.

Rolf Harbeck

Unserem Gasteditor zu diesem Heft dankt die Schriftleitung der KN recht herzlich für sein Engagement und die ehrenamtliche Ausübung dieser Funktion! 